\title{
Processing strategies in picture-sentence verification tasks
}

\author{
JILL T. WANNEMACHER \\ Bryn Mawr College, Bryn Mawr, Pennsylvania 19010
}

\begin{abstract}
The encoding and comparison strategies used by observers in matching pictures and sentences were investigated. The location of the mismatch or difference between a sentence and a picture was varied, and the latencies of "same"."different" responses were compared as a function of whether the mismatch occurred in the sentence subject, verb, or object. Sentences were presented auditorily and varied in both voice and reversibility. Pictures either preceded or were presented simultaneously with the sentences. In all cases, Ss adopted a serial self-terminating comparison strategy and a surface structure encoding strategy: The comparison was terminated as soon as a mismatch was encountered, and actives were processed in the order subject-verb-object, whereas passives were processed in the order object-verb-subject.
\end{abstract}

Most current theories of sentence processing view comprehension not as a global, unitary process, but rather as a sequence of independent, serial mental operations, each of which has real-time characteristics. Thus, in tasks such as picture-sentence matching, the decision as to whether a sentence is an accurate description of a picture is thought to require a processing sequence such as the following: (1) encoding the stimuli, (2) comparing the two stimulus encodings, and (3) formulating an appropriate response decision (e.g., Clark, 1973; Clark \& Chase, 1972; Olson \& Filby, 1972; Trabasso, 1972). The present experiments sought to determine the nature of the encoding and comparison strategies used by Ss in matching pictures and sentences, as well as to determine the stages in the processing sequence that are influenced by such variables as sentence voice and reversibility.

These studies differ from previous experiments that have used a verification paradigm in that a novel procedure for independently manipulating the duration of the comparison process was utilized. This procedure, called the "mismatch" technique, involved varying the location of the mismatch or difference between a sentence and a picture and comparing the latencies of the Ss" "same"-"different" responses as a function of the position of the mismatch, that is, as a function of whether it occurred in the sentence subject, verb, or object. A "same" response, thus, always required three specific comparisons. In the case of a "different" response, however, the number of comparisons that was sufficient for a response decision varied with the location of the mismatch: Subject mismatches required only one comparison, verb mismatches required two, and object mismatches required three, given a serial

*I am indebted to Peter D. Eimas for his careful and thoughtful criticisms of this research, this paper, and the doctoral dissertation on which it is based (Brown University, 1973). I also wish to thank the consulting editor and Howard S. 1973). I also wish to thank the consulting editor and Howard Hoffman for their helpful advice regarding an earlier version of
this paper. This research was supported by PHS Grant HD 05531 to Peter D. Eimas from the National Institutes of Child Health and Human Development. self-terminating comparison process and a subject-verbobject order of comparison.

The specific issues which this technique is capable of clarifying include: (1) how sentences are encoded, that is, the format in which linguistic information is represented;(2) how sentences are processed, e.g., whether the comparison strategy adopted by $S s$ in picture-sentence matching tasks is serial or parallel, exhaustive or self-terminating, and whether such strategies change with the demands of the task; and (3) what stages in the processing sequence (e.g., encoding, comparison, response decision) are influenced by variables such as sentence voice and reversibility. These issues are very similar to those which Sternberg (1969) dealt with in his studies of memory retrieval. His success in delineating the stages, underlying processes, and factor-stage relations in memory retrieval tasks was due primarily to his ability to vary the duration of the comparison stage independently of the duration of other stages such as encoding. Thus, by varying the number of stimulus elements in memory, Stemberg could effectively control the number of comparisons that was required, and in this manner he was able not only to determine the type of comparison strategy adopted by Ss within the comparison stage (e.g., serial exhaustive) but also to specify the stages influenced by variables such as stimulus degradation, since whether a stimulus was intact or degraded did not change the number of comparisons that was required.

The mismatch technique used in the present studies provides a method analogous to Sternberg's for independently manipulating the duration of the comparison stage in verification tasks, in this case by varying the number of comparisons that must be made between a sentence and a picture before a match-mismatch decision can be reached. By examining the differences in reaction time (RT) to subject, verb, and object mismatches, it should be possible to determine both the type of comparison strategy that is used and the nature of the encoding format. For 
example, if Ss adopt a serial self-terminating comparison strategy in the order subject-verb-object, RTs to active sentences with a subject mismatch should be fastest, and whether the verb and/or object also mismatch should have no effect. Such a response pattern would not rule out a parallel self-terminating strategy, although a consistent increase in RT from subject to verb to object mismatches would favor a serial interpretation. If, on the other hand, search is exhaustive, there should be no systematic variation in RT as a function of the serial position of the mismatch. In addition, by examining the pattern of RTs to the various mismatch types in both active and passive sentences, it should be possible to determine whether sentences are encoded in terms of their underlying propositions, as Clark (1973) would suggest, or whether they are in fact processed in their surface structure formats, as indicated by the results of Olson and Filby (1972): In passive sentences the superficial (surface structure) order of the lexical constituents is (1) logical (deep structure) object, (2) verb, (3) logical subject. Thus, if a passive sentence is encoded in its surface structure format (i.e., in the order object verb subject), then RTs to sentences with object mismatches should be faster than RTs to sentences with subject mismatches, assuming a serial self-terminating processing strategy.

The technique also provides a means of investigating the relationship between syntactic and semantic variables in sentence processing, for example, whether they influence the same or different stages and whether they affect one, or more than one, stage in the processing sequence. Sentences were presented in either the active or the passive voice (syntactic complexity), and the subject and object of a particular sentence were either semantically reversible or nonreversible, that is, the subject and object could logically be switched (e.g., "the boy is kissing the girl") or not (e.g., "the boy is watering the grass"). Voice and reversibility have previously been found to interact in verification tasks (e.g., Slobin, 1966): Passives take longer than actives when sentences are reversible but are processed as quickly as actives when sentences are nonreversible, presumably because there is no possibility of confusing the subject and object of action. Trabasso (1972) and Bever (1970) have suggested that both voice and reversibility influence the encoding stage. If this is indeed the case, then in the present studies neither variable should have a differential effect on processing as a function of the number of comparisons required in the comparison stage, assuming the encoding and comparison stages are independent.

\section{METHOD}

\section{Subjects}

The Ss in each study were seven students in the introductory psychology course at Brown University. Each participated in two experimental sessions lasting approximately $1 \mathrm{~h}$ and was paid a total of $\$ 4$.
Stimuli

The sentences were of four types: (1) active reversible (AR), e.g., "the horse is biting the cow"; (2) active nonreversible (ANR), e.g., "the boy is watering the grass"; (3) passive reversible (PR), e.g., "the cow is being bitten by the horse"; (4) passive nonreversible (PNR), e.g., "the grass is being watered by the boy." The number of syllables ranged from 7 to 9 in active sentences and from 9 to 12 in passive sentences. The subjects and objects for reversible sentences were chosen from the categories "human," "animal," and "vehicle," with any plausible and potentially reversible combination of nouns from the three categories being permitted. Sentences such as "the puppy is licking the boy" were not used because its reversed counterpart, while possible, is not plausible. The subject and object of every reversible sentence thus could logically be reversed, although no false sentence was constructed by in fact reversing the subject and object. The word substitutions for the mismatching sentences were selected so as to avoid any ambiguity. For example, "boy" was replaced by a noun of the opposite sex, such as "girl," and not "man," "dog" was replaced by "cat" and not "wolf," and "kicking" was replaced by "biting" and not "hitting." The subjects for nonreversible sentences were primarily from the category "human," and only verbs that are always nonreversible were included. The objects in nonreversible sentences were of necessity all inanimate nouns, and again the substitutes were always as distinctive as possible (e.g., "apple" was replaced by "banana" and not "orange").

The pictures were colored cartoon-like drawings that were photographed and mounted in to $35-\mathrm{mm}$ slides.

\section{Design}

The three independent variables manipulated within Ss were voice (active or passive), reversibility (reversible or nonreversible), and treatments (true sentences and seven mismatch, or false, types). Each $S$ was presented with a total of 280 sentences, representing 70 of each of the four types-AR, ANR, PR, PNR. Within each group of 70, 35 of the sentences matched the picture and 35 mismatched. The 35 mismatching sentences of each type consisted of five instances of each of seven mismatch types: subject (s), verb (v), object (o), subject-verb (s-v), verb-object (v-o), subject-object (s-o), and subject-verb-object (s-v-o). The sentences were grouped into five blocks of 56, each containing an equal number of matches and mismatches and an equal number of each sentence type. The order of sentences within each block was random. Within Ss each individual picture was paired with only one type of mismatching sentence (e.g., only with an s mismatch), but across Ss each picture was paired with a different mismatch type. Thus, across Ss each particular picture was paired with every possible type of mismatching sentence. Finally, within Ss each of the 70 pictures was viewed four times, paired with (1) a true active sentence, (2) a true passive sentence, (3) a false active sentence, and (4) a false passive sentence.

\section{Apparatus}

The pictures (35-mm slides) were presented by means of a random-access projector and projected onto a half-silver glass screen in front of the $S$. The projected size of the pictures was approximately $7 \times 9$ in. The sentences were tape recorded on Channel 1 of an Ampex tape recorder and presented binaurally by means of headphones worn by both the $S$ and $E$. The RT was measured from the first sentence noun: Noise signals inserted after the first syllable of the noun on Channel 2 activated a voice key, which in turn started a clock. The duration of the picture was controlled by a Hunter timer. The S's pushbutton response stopped the clock and lit one of two indicator lights corresponding to either a "same" or a "different" response. The RT was measured to the nearest millisecond.

\section{General Procedure}

Session 1 consisted of 13 practice trials followed by Stimulus 
Fig. 1. Mean RT to true sentences and seven types of mismatching sentence in Experiment I. The RTs for active reversible (AR) and nonreversible (ANR) sentences are in $\mathrm{Fig} .1 \mathrm{~A}$, and those for passive reversible (PR) and nonreversible (PNR) sentences are in Fig. 1B. Mismatch types refer to the deep structure functions of the lexical constituents. The range of the standard error of the mean was 39 to $90 \mathrm{msec}$ in AR sentences, 40 to $105 \mathrm{msec}$ in ANR sentences, 47 to $112 \mathrm{msec}$ in PR sentences, and 39 to $104 \mathrm{msec}$ in PNR sentences.
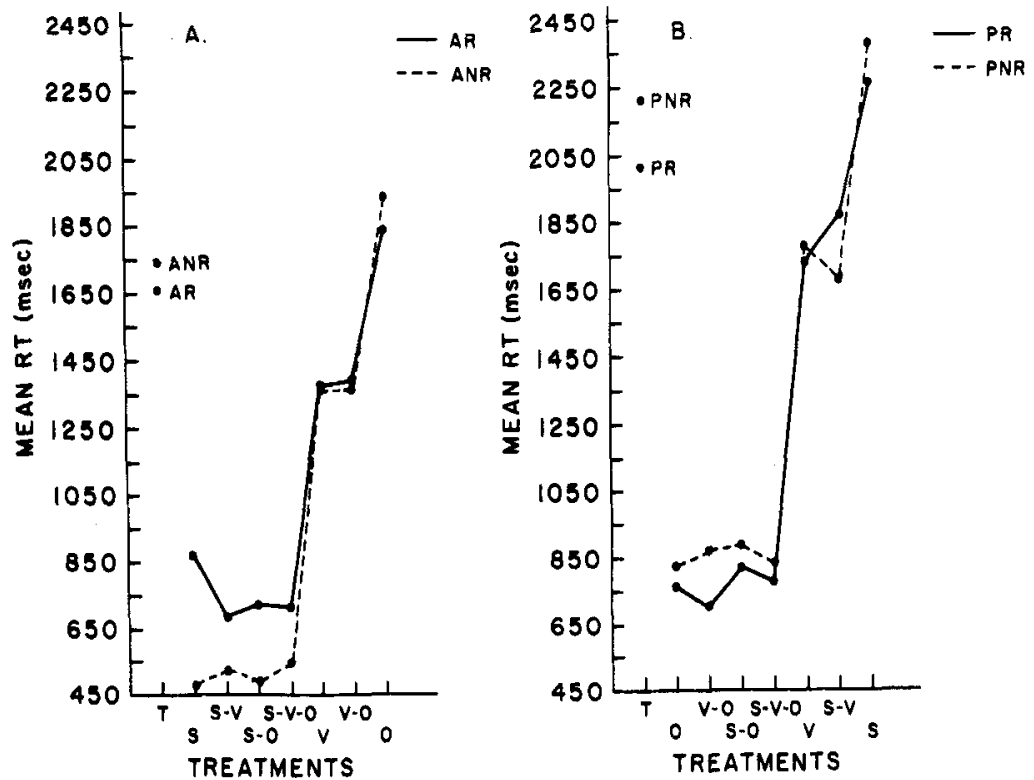

Blocks 1 and 2. Stimulus Blocks 3, 4, and 5 were administered in Session 2. The duration of each block was approximately $20 \mathrm{~min}$, and each was followed by a 5-min rest interval. Errors were corrected during the practice trials, but Ss received no feedback regarding the correctness of their responses during the experimental trials.

The Ss were told to decide whether the sentence matched the picture and to respond by depressing one of two pushbuttons labeled "same" and "different." The instructions placed equal emphasis on speed and accuracy. The pairing of response hand to "same" and "different" was varied by initially asking each S whether his preferred hand was left or right, then instructing alternate Ss to respond "same" with their preferred hand.

\section{EXPERIMENT I}

\section{Pictures Preceding Sentences}

Immediately following a verbal "ready" signal, a picture was presented and remained available for viewing for $2 \mathrm{sec}$. Offset of the picture was followed by a second "ready" signal, a 1-sec pause, then onset of the sentence. The RT was measured from the offset of the first syllable of the first noun in the sentence to allow for the possibility that Ss might respond before the end of the sentence if sufficient information for a mismatch decision had been presented.

\section{Results and Discussion}

Errors. The range of errors for individual Ss was from 5 to 13 , with a mean of 9.9 . The total number of errors was 69 , or $3.5 \%$. Fifty of these were incorrect "false" responses, or errors in which true sentences were called "false," and RTs were as slow as correct responses to true sentences. Only correct responses were included in the RT analyses.

The Processing Strategy. Mean RT to each treatment type (true and seven mismatch types) is shown in Fig. $1 \mathrm{~A}$ for active reversible and nonreversible sentences and in Fig. 1B for passive reversible and nonreversible sentences. The labeling of the mismatch types on the abscissas refers to the deep structure function of the lexical constituents. Thus, a mismatch in the first noun of active sentences ( $\mathrm{s}, \mathrm{s}-\mathrm{v}, \mathrm{s}-\mathrm{o}, \mathrm{s}-\mathrm{v}-\mathrm{o}$ ) involved the deep structure (logical) subject, while a mismatch in the first noun of passive sentences ( $\mathrm{o}, \mathrm{v}-\mathrm{o}, \mathrm{s}-\mathrm{o}, \mathrm{s}-\mathrm{v}-\mathrm{o})$ involved the deep structure (logical) object. The similarity of the functions for actives and passives indicates the same processing strategy was used in both cases, but active sentences were processed in the order subject-verbobject, while passives were processed in the order objectverb-subject. In other words, passive sentences were not transformed into an active format prior to comparing them with the pictures.

A four-way analysis of variance (Ss by voice by reversibility by treatments) was performed on the mean RTs, with the appropriate Ss by Effects interactions serving as denominators for each $F$ ratio. The effect of treatments was significant beyond the .001 level $[F(7,42)=199.71]$, and subsequent Newman-Keuls analyses revealed the same pattern of significant differences $(p<.01)$ for each sentence type $(A R, A N R$, PR, PNR): (1) All mismatches involving the first noun of the sentence (subject in actives and object in passives) were processed equally fast; mean RTs (milliseconds) for the four sentence types were 730 (AR), 514 (ANR), 738 (PR), and 788 (PNR); (2) the two mismatch types involving the verb were also processed equally fast (mean RTs: $\mathrm{AR}=1388, \mathrm{ANR}=1365, \mathrm{PR}=2266$, PNR $=1721$ ), but they took significantly longer than mismatches in the first noun; (3) the RTs to mismatches involving the object in actives and the subject in passives were significantly longer than RTs to any other mismatch type (mean RTs: $A R=1836, A N R=1938$, $\mathrm{PR}=2266, \mathrm{PNR}=2378)$, but equaled the RTs to true sentences (mean RTs: $\mathrm{AR}=1657, \quad \mathrm{ANR}=1737$, $P R=2025, P N R=2218$ ). The data, thus, fulfill all of the predictions of a serial self-terminating model.

Voice was also a significant main effect, despite the 
fact that the interactive effect of voice with treatments was removed by analyzing the mean RTs in terms of the surface structure location of the mismatch. The effect of voice is largely due to the difference in sentence length between actives and passives, since RT was of necessity measured from the beginning of the sentence: Figure 1 shows that RTs to verb and object mismatches and true sentences were longer in passives than in actives. However, there were also significant interactions between (1) voice and reversibility $[\mathrm{F}(1,6)=10.67$, $\mathrm{p}<.05]$, (2) voice and treatments $[\mathrm{F}(7,42)=5.46$, $p<.01]$, and (3) voice, reversibility, and treatments $[F(7,42)=2.35, p<.05]$ which cannot be attributed to differences in sentence length. The interactions all reflect the fact that mismatches in the first noun of ANR sentences were processed faster than the corresponding mismatches in the other three sentence types. A post hoc interpretation of this finding is that it is due to a lower relative uncertainty associated with mismatches in the first noun of ANR sentences.

Specifically, in ANR sentences only the first noun (subject or agent) is animate; thus, if the first noun the $S$ hears is animate and it does not match the agent of the picture, he can respond "false" very quickly, since there is no possibility that the sentence is passive and that the first noun is in fact the object. In reversible sentences, both active and passive, the subject and object are both either animate or vehicles, and the two could logically be reversed; a response decision for a mismatch in the first noun thus takes slightly longer, because the first noun could be either the agent or the object of the sentence. It should be noted, however, that Ss do not wait to process the verb before responding, since RTs to verb mismatches were significantly longer than RTs to mismatches in the first noun. Finally, in the case of PNR sentences, RTs to mismatches in the first noun may be slower because they involve inanimate objects, which are less salient and have more potential alternatives than an animate noun. Nevertheless, the results in Figure 1 clearly indicate that passive sentences were processed in their surface structure (object-verb-subject) formats and that Ss used a serial self-terminating strategy in comparing the two stimulus inputs.

Factor-Stage Relations in Processing. The results of Experiment I pose two major problems for processing models such as the one proposed by Clark (1973). First, they indicate that, when sentences are presented auditorily and follow the pictures, they are not represented in terms of their underlying or deep structure propositions; the sentence constituents are processed in their surface structure order. Second, the results suggest that the sequence of processing stages-encoding, comparison, and response decision-is applied to each lexical constituent individually rather than operating on the sentence as a whole. The finding that Ss responded before the end of the sentence when there was a mismatch in either the first noun or the verb indicates the unit of processing was not the entire sentence but rather the individual constituent, that is, subject, verb, object.

Such an interpretation requires a serial stage analysis which is similar to that proposed by Clark but more accurately describes the processing sequence that occurs when Ss adopt a surface structure encoding strategy and a serial self-terminating comparison strategy. Specifically, in such situations Ss are not assumed to encode the entire sentence prior to beginning comparison operations. Instead, the information processing sequence is applied to the separate sentence constituents consecutively, in a series of stages as follows: encode first noun; compare first noun against picture; if mismatch, exit and respond false; if match, encode verb, compare verb against picture, and so on. The encoding, comparison, and response decision stages can, thus, be considered a processing loop, and the number of sentence constituents to which it is applied depends on whether the sentence is true or false and, if false, on the location of the mismatch.

Given such a view of the processing sequence, the factor-stage relations in Experiment I can be delineated as follows: (1) Voice determined the order in which the sentence constituents were processed; in active sentences the processing loop operated on the constituents in the order logical-subject verb logical-object, while in passives the order of processing was logical-object verb logical-subject. (2) Treatments determined the number of sentence constituents to which the processing loop was applied before the $S$ could exit the sequence and, hence, the duration of processing; only one application was required when mismatches occurred in the first noun, verb mismatches required two, and true sentences and mismatches in the second noun required three. (3) Reversibility was not a significant main effect, but the nature of its interactions with voice and treatments indicates it affected the duration of the response decision process, that is, the speed of making a decision to respond "false." This is suggested by the finding that RTs to mismatches in the first noun were faster in ANR sentences than in the other three sentence types: Processing was terminated in both reversible and nonreversible sentences when a mismatch occurred in the first noun, since RTs were always significantly faster than those to verb mismatches; however, in the case of ANRs, the decision to respond "false" to a mismatch in the first noun could be made more quickly than in the other three sentence types. The effect of reversibility was, thus, limited to the response decision stage of the first processing loop in ANR sentences. This did not, however, speed up processing of the verb and object in ANR sentences without subject mismatches, since the auditory presentation of sentences made processing rate contingent upon presentation rate (approximately two words per second).

\section{EXPERIMENT II}

\section{Simultaneous Presentation of Sentences and Pictures}

Experiment II differed from Experiment I only in 
Fig. 2. Mean RT to true sentences and seven types of mismatching sentence in Experiment II. The RTs for active reversible (AR) and nonreversible (ANR) sentences are in Fig. 2A, and those for passive reversible (PR) and nonreversible (PNR) sentences are in Fig. 2B. Mismatch types refer to the deep structure functions of the lexical constituents. The range of the standard error of the mean was 48 to $166 \mathrm{msec}$ in AR sentences, 45 to $138 \mathrm{msec}$ in ANR sentences, 54 to $160 \mathrm{msec}$ in PR sentences, and 34 to $128 \mathrm{msec}$ in PNR sentences.
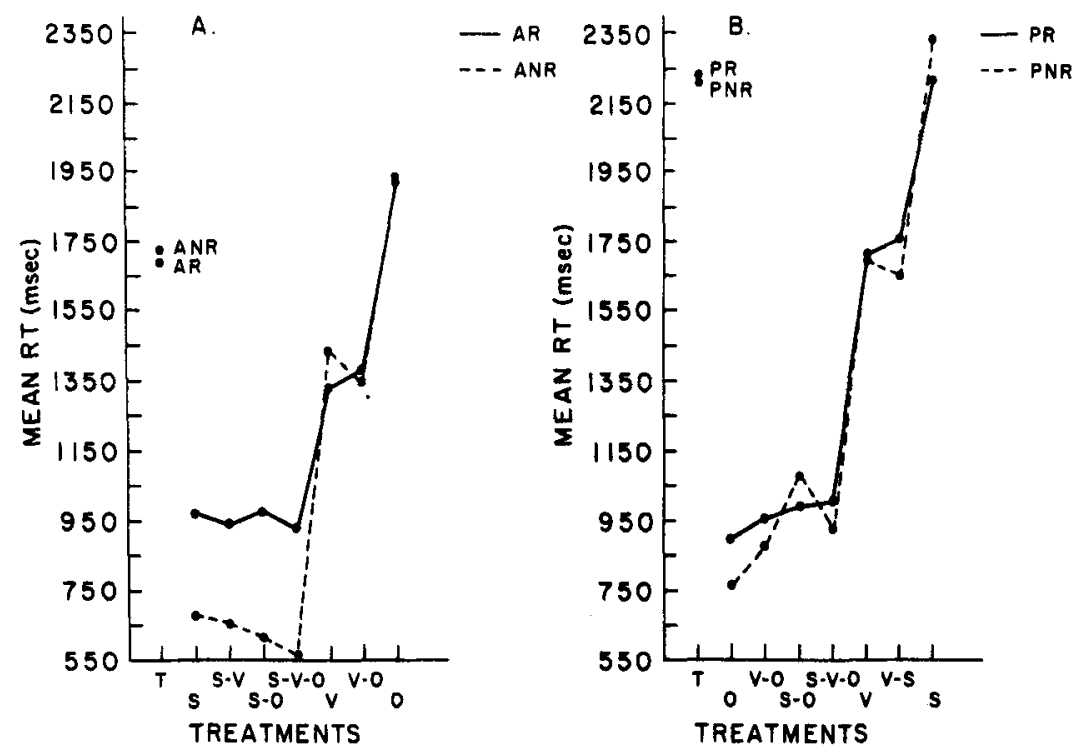

that the sentences and pictures were presented simultaneously. A verbal "ready" signal was followed after a 1 -sec pause by onset of both the sentence and the picture. The RT was measured from the beginning of the first sentence noun. The picture remained available for viewing until after the S's response or for a maximum of $3 \mathrm{sec}$, whichever came first.

\section{Results and Discussion}

Errors. The range of errors for individual Ss was from 6 to 19 , with a mean of 10.4 . The total number of errors was 73 , or $3.7 \%$, and 48 of these were incorrect "false" responses. The RTs on 43 of the 48 incorrect "false" responses were as slow as or slower than correct responses to true sentences. Only correct responses were included in the RT analyses.

The Processing Strategy. Mean RTs to true sentences and each of the seven mismatch types in Experiment II are shown in Figs. 2A and 2B. An in Experiment I, the Ss adopted a serial self-terminating strategy in comparing the sentences and pictures, regardless of voice or reversibility. Again, however, sentences were processed in their surface structure formats: The order of comparison was subject-verb-object for active sentences and object-verb-subject for passive sentences.

A four-way analysis of variance (Ss by voice by reversibility by treatments) showed significant main effects of voice $[F(1,6)=54.35, p<.001]$, reversibility $[F(1,6)=18.28, p<.01]$, and treatments $[F(7,42)=150.41, p<.001]$, as well as significant interactions between voice and reversibility $[F(1,6)=11.64, p<.025]$, voice and treatments $[F(7,42)=5.78, p<.01]$, reversibility and treatments $[F(7,42)=4.14, p<.01]$, and voice, reversibility, and treatments $[F(7,42)=2.96, p<.05]$. Newman-Keuls analyses of the treatments effect revealed the same pattern of significant differences between treatments as in Experiment I with two exceptions: (1) In AR sentences, o mismatches were significantly slower than true responses, and (2) in PNR sentences, s-o mismatches were significantly slower than o mismatches. With regard to the former, it should be noted that in both Experiments I and II RTs to true sentences tended to be faster than RTs to the slowest mismatch type, although this was the only instance in which the difference was significant. This finding suggests that Ss are "set" to make a "true" response and that a mismatch encountered at the end of the sentence necessitates some process equivalent to the resetting of a "truth index," as proposed in the models developed by Clark (1973), Clark and Chase (1972), Olson and Filby (1972), and Trabasso (1972). The relatively long RT to s-o mismatches in PRN sentences apparently resulted because on a small number of trials Ss failed to detect the $o$ mismatch (an inanimate object) at the beginning of the sentence but did respond to the $s$ mismatch at the end. As a result, several long RTs corresponding to the time to detect an $\mathrm{s}$ mismatch were averaged in with the RTs to s-o mismatches and RT was raised.

The effect of voice and its interaction with treatments is again largely due to the difference in sentence length between actives and passives, at least in the case of mismatches in the verb and final noun. The effect of reversibility, its interactions with voice and treatments, and the three-way interaction are best understood by examining the RTs to mismatches involving the first noun in Figs. 2A and 2B: RTs to mismatches in the first noun were faster in nonreversible than in reversible sentences, and this difference was greater for actives than for passives; mean RT to the four mismatch types involving the subject was $956 \mathrm{msec}$ in AR sentences vs $624 \mathrm{msec}$ in ANR sentences, and mean RT to the four mismatch types involving the object was $966 \mathrm{msec}$ in PR sentences vs $920 \mathrm{msec}$ in PNR sentences. As in Experiment I, the finding that RTs to subject mismatches in ANR sentences are extremely fast is 
Table 1

Mean RTs (Milliseconds) to Mismatches in the First Sentence Noun

\begin{tabular}{lccc}
\hline & \multicolumn{2}{c}{ Experiment } & \\
\cline { 2 - 3 } $\begin{array}{l}\text { Sentence } \\
\text { Type }\end{array}$ & I & II & Difference \\
\hline AR & 750 & 956 & 206 \\
PR & 767 & 967 & 200 \\
ANR & 511 & 624 & 113 \\
PNR & 853 & 923 & 70 \\
\hline
\end{tabular}

apparently due to the lower relative uncertainty associated with a mismatch in the first noun in ANR sentences.

Factor-Stage Relations in Processing. The factor-stage relations in Experiment II are very similar to those in Experiment I, since the simultaneous presentation of sentences and pictures did not alter the Ss' processing strategy. Again, therefore, the information processing sequence can best be represented as a processing loop, consisting of an encoding, a comparison, and a response decision stage applied to each lexical constituent individually. Sentence voice determined the order in which the sentence constituents were processed; the sequence for actives was logical-subject verb logical-object, while that for passives was logical-object verb logical-subject. Treatments determined the number of times the processing loop was applied-once when a mismatch occurred in the first noun, twice for verb mismatches, and three times when sentences were true or a mismatch occurred in the second noun. Reversibility again influenced the response decision stage of the first processing loop in ANR sentences, with "false" responses to mismatches in the first noun being made more quickly than in the other three sentence types.

Experiment II differed from Experiment I in that RT included picture encoding time, which was not the case when pictures preceded sentences. A consideration of the nature of the two stimulus inputs suggests that, whereas the three sentence constituents (because of their serial presentation) were encoded separately in distinct processing loops, the parallel presentation of the picture constituents probably resulted in a single picture encoding stage, which overlapped the processing of the first sentence noun. If this were the case, mean RTs to the four mismatch types involving the first noun in Experiment II should exceed those in Experiment I, since the former include picture encoding time, while the latter do not. An examination of the data in Figs. 1 and 2 reveals such a difference: The mean RT to mismatches in the first noun (across all four sentence types) was $175 \mathrm{msec}$ longer in Experiment II than in Experiment I (867 vs 692), while means RTs to verb mismatches (1543 vs 1567$)$, object mismatches (2103 vs 2106 ), and true sentences (1969 vs 1909) were very similar.

A breakdown of the mean RTs to first-noun mismatches in each sentence type reveals that the duration of picture encoding was influenced by reversibility. Table 1 shows the mean RTs obtained in Experiments I and II and the differences between them. The specific numerical differences are not a reliable measure of the duration of encoding, since the data are from two different experiments, but the magnitude of the difference between the time for reversibles and that for nonreversibles does indicate that reversible pictures are encoded more slowly than nonreversible pictures. It should also be noted that the inequality in the "encoding" times for active and passive nonreversibles (113 vs $70 \mathrm{msec}$ ) supports the assumption that picture encoding and the processing of the first sentence noun overlapped in Experiment II: The uncertainty associated with an object mismatch in PNR sentence-picture pairs was apparently less in Experiment II than in Experiment I, possibly because the name of the inanimate object (the first noun) was being presented as the picture was being encoded.

\section{GENERAL DISCUSSION}

The results of Experiments I and II clearly indicate Ss adopted a serial self-terminating strategy in comparing the lexical constituents of sentences and pictures: Mismatches occurring in the first noun were processed fastest, followed by verb mismatches; slowest RTs were to true sentences and mismatches occurring at the end of the sentence, both of which required three comparisons. The same processing strategy was used for both active and passive sentences, but since passives were encoded in their surface structure formats, the order of comparison was object-verb-subject, while that for actives was subject-verb-object. The auditory presentation of sentences, which most closely resembles the natural language processing situation, undoubtedly maximized the efficiency of both a serial self-terminating comparison strategy and a surface structure encoding strategy. It remains to be determined whether the same strategies are adopted when sentences precede rather than follow the pictures or when the Ss are children rather than adults. It should be noted, however, that Glucksberg, Trabasso, and Wald (1973), in a recently reported study, utilized a similar mismatch procedure in verification tasks where sentences were presented visually and obtained quite different results: Ss apparently did not process passives in their surface structure formats, and the results indicated the comparison process was not initiated until after the entire sentence had been encoded, a finding which attests to the flexibility of human Ss in information processing situations.

With regard to the factor-stage relations in processing, the results of the present studies pose several problems for models such as the one proposed by Clark (1973). Sentences presented auditorily rather than visually are not necessarily represented in terms of their underlying or deep structure propositions. Furthermore, if Ss adopt 
a serial self-terminating processing strategy, the individual sentence constituent rather than the entire sentence becomes the unit of processing. The results of the present studies are best accommodated by an information processing strategy that consists of the same serial stage sequence as that proposed by Clark, but operating as a processing loop on the discrete sentence constituents rather than on an encoded representation of the entire sentence. In addition, the effects of a particular variable need not be limited to a single stage in the processing sequence. For example, in Experiment II reversibility apparently influenced both a response decision stage and the duration of picture encoding. Finally, when sentences are encoded in their surface structure formats, the factor-stage relations observed differ from those predicted by a model that assumes processing operates on the underlying propositions of stimuli: In the present studies, sentence voice did not influence the duration of the comparison process, as proposed by Clark's model, but rather the order in which the comparisons were initiated.

The model suggested by Olson and Filby (1972) does assume passives are encoded in their surface structure formats, but its ability to specify the details of the comparison operation is limited, as in Clark's model, by the paucity of the data available to date on the strategies used by $\mathrm{Ss}$ in verification tasks. By utilizing procedures such as the mismatch technique in a variety of tasks and systematically varying the task demands, it should be possible to describe the strategies adopted by $S$ in different situations and to delineate the factor-stage relations that occur as the task demands are changed. In all likelihood, no single specific model will apply in all picture-sentence verification tasks; human $\mathrm{Ss}$ are extremely flexible in their approach to information processing and can modify their encoding and comparison strategies as the task demands are changed in order to achieve maximum efficiency.

\section{REFERENCES}

Bever, T. G. The cognitive bases for linguistic structures. In J. R. Hayes (Ed.), Cognition and the development of language. New York: Wiley, 1970.

Clark. H. H. Semantics and comprehension. In T. A. Sebeok (Ed.), Current trends in linguistics, Vol, 12: Linguistics and adjacent arts and gciences. The Hague: Mouton, 1973.

Clark, H. H., \& Chase, W. G. On the process of comparing sentences against pictures. Cognitive Psychology, 1972, 3, $472-517$.

Glucksberg, S., Trabasso, T., \& Wald, J. Linguistic structures and mental operations. Cognitive Psychology, 1973, 5, 338-370.

Olson, D. R. \& Filby, N. On the comprehension of active and passive sentences. Cognitive Psychology, 1972, 3, 361-381.

Slobin, D. I. Grammatical transformations and sentence comprehension in childhood and adulthood. Journal of Verbal Learning \& Verbal Behavior, 1966, 5, 219-227.

Sternberg, $S$. The discovery of processing stages: Extensions of Donders' method. Acta Psychologica, 1969, 30, 276-315.

Trabasso, T. Mental operations in language comprehension. In $J$. B. Carroll and R. O. Freedle (Eds.), Language comprehension and the acquisition of knowledge. Washington, D.C: Winston, 1972.

(Received for publication October 29,1973 ; revision received January 21,1974 .) 